\section{March 2000}

British Orthodontic

Society: Faces, Braces

and Spaces

Date: 18.03 .00

Venue: London

Contact: BOS Office

Eastman Dental Hospital

256 Gray's Inn Road, London WC1X 8LD

Tel/Fax: +44 (0)20 78372193

HC2000: Healthcare Computing

Conference \& Exhibition

Date: $20.03 .00-22.03 .00$

Venue: Harrogate

Contact: Fiona Campbell, BJHC,

45 Woodland Grove,

Weybridge,

Surrey KT13 9EQ

Tel: +44 (0) 1932821723

Fax:+44 (0)1932 820305

Website: www.healthcare-computing.co.uk

The 2000 Meeting Of The Craniofacial

Society Of Great Britain

Date: 22.03.00 - 24.03.00

Venue: Reading

Contact: Congress Secretariat

Craniofacial Society of Great Britain

The Royal College of Surgeons

35-43 Lincoln's Inn Fields

London WC2A 3PN

Tel: +44 (0)20 74044785

Fax: +44 (0)20 78314041

E-mail: secretariat@craniofacial.co.uk

London 2000: International

Congress on Implant

Dentistry

Date: $31.03 .00-01.04 .00$

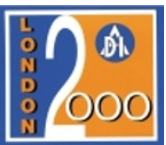

Venue: London Heathrow Marriott Hotel

Contact: Cherry Wilson,

Association of Dental Implantology UK

37 Halford Road, Richmond,

Surrey TW10 6AW

Tel: +44 (0)20 83320321

Fax: +44(0)20 89400337

E-mail: adi.uk@btinternet.com

Website: www.adi.org.uk

\section{April 2000}

BDA Conference: Dentistry 2000 - The Millennium celebration for oral care Date: 06.04 .00 - 08.04 .00

Venue: Birmingham

Contact: Charlotte Long,

BDA Conference Office,

64 Wimpole Street, London W1M 8AL

Tel: +44 (0)20 79350875 ext 286

Fax: +44(0)2074860855

Setting Up A Practice Seminar

Date: 07.04 .00 and 24.06.00

Venue: Birmingham and London

Contact: Sarah Bradbury/Martin Cock,
British Dental Association,

64 Wimpole Street, London W1M 8AL

Tel: +44 (0)20 79350875 ext 260

Fax: +44 (0)2074860855

DPB Dental Conference and Open

Meeting

Date: 17.4 .00 - 18.4 .00

Venue: Eastbourne

Contact: Heather Deacon

Tel: +44 (0) 1323433307

Fax:+44 (0) 1323433432

E-mail: pr@dentanet.org.uk

\section{May 2000}

9th International Dental Congress On Modern Pain Control: A new millennium in analgesia, sedation and anaesthesia Date: 02.05.00 - 05.05.00

Venue: Jersualem, Israel

Contact: 9th IFDAS Secretariat,

International Travel and Congresses Ltd, P.O. Box 29313,

20 Rothschild Boulevard,

Tel Aviv 61292, Israel

Tel: +972 37951444

Fax: +97235107716

E-mail: congs@internationaltc.co.il

Website: www6.huji.ac.il/ dental/news.htm

The British Society for Restorative Dentistry Spring Scientific Meeting: Bright ideas for the 21st Century

Date: $05.05 .00-06.05 .00$

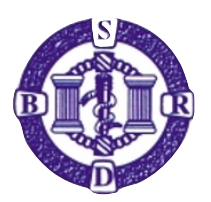

Venue: Windsor

Contact: Janet Harlow

Tel: +44 (0)1612756797

Website: Janet.Harlow@man.ac.uk

\section{CONSEURO 2000}

Date: 11.05 .00 - 13.05.00

Venue: Bologna, Italy

Contact: Dr Margaret Wilson,

Restorative Dentistry,

University Dental Hospital of Manchester,

Higher Cambridge Street,

Manchester M15 6FH.

Tel: +44 (0)1612756619

Fax: +44 (0)1612756710

E-mail: wilson@fs1.den.man.ac.uk

\section{June 2000}

British Association for Cancer Research, Head and Neck Oncology Workshop

Date: 30.06 .00

Venue: London

Contact: Dr Max Partridge

Maxillofacial Unit/Oncology

King's College Hospital

Denmark Hill

London SE5 8RX

Tel: +44 (0)2073463474

Fax: +44 (0)2073463754

E-mail: max.partridge@kcl.ac.uk
August 2000

The FDI's Jubilee Conference, London

Date: 15.08 .00

Venue: London

Contact: FDI

7 Carlisle Street

London

Tel: +44 (0)20 79357852

Fax: +44 (0)20 74860183

E-mail: congress@fdi.org.uk

Internet: www.fdi.org.uk

\section{October 2000}

New Zealand Dental Association Biennial Conference

Date: 04.10 .00 - 08.10 .00

Venue: Dunedin, New Zealand

Contact: NZDA Conference,

PO Box 1029, Dunedin, New Zealand

Tel: +64 34771377

Fax: +64 34772720

E-mail:dcms@dcms.co.nz

November 2000

FDI World Dental

Congress 2000

Date: $29.11 .00-02.12 .00$

Venue: Paris

Contact: FDI, 7 Carlisle

Street, London W1V 5RG

Tel: +44 (0)20 79357852

Fax: +44 (0)20 74860183

Website: www.fdi.org.uk

International Dental

Film Festival

Date: $29.11 .00-02.12 .00$

Venue: Paris

Contact: Association Dentaire Francaise,

6 Rue Guillaume Tell 75017, Paris.

Tel: +33144010270

Fax: +33147639028

E-mail: adf@adf.asso.fr

Website: www.adf.asso.fr

\section{1}

\section{May 2001}

30th Australian Dental Congress

Date: 04.05.01 - 08.05.01

Venue: Brisbane, Australia

Contact: Secretariat, 30th Australian

Dental Congress, P.O. Box 1280 Milton,

Queensland 4064, Australia

Tel: +61 733690477

Fax: +61 733691512

E-mail:ada2001@im.com.au

Please send event details to:

Kate Craig, BDJ

64 Wimpole Street, London WIM 8AL, UK 